\title{
EVALUATION OF THE DIMENSION OF THE Q-VECTOR SPACE SPANNED BY THE SPECIAL VALUES OF THE LERCH FUNCTION
}

By

\author{
Makoto KawASHIMA
}

\begin{abstract}
Nikisin [6] proved the linear independence of special values of polylogarithm functions over the rational number field. (see Theorem 0.1). In this paper, we give a generalization of the above result of Nikisin to the case of the Lerch function.
\end{abstract}

\section{Introduction}

A lot of problems on special values of the Riemann zeta function at positive integers, for example, irrationality or trancendency of these numbers, remain unsolved in many cases. The Padé approximation of the polylogarithm functions and the Hurwitz zeta function are one of the main methods to study these problems and have been studied for the polylogarithm functions (see [1], [3], [4], [6], [9], [10]) and for the Hurwitz zeta function (see [2], [8]). We recall the following result of Nikisin which is one of the classical results for the Padé approximation of the polylogarithm functions.

Theorem 0.1 ([6, Theorem 1]). Let $s \in \mathbf{N}$ and $\operatorname{Li}_{s}(z)=\sum_{n=1}^{\infty} \frac{z^{n}}{n^{s}}$ be the $s$-th polylogarithm function. Let $z$ be a negative rational number and assume, for unique coprime integers $a, b$ such that $z=a / b$ with $b>0$ and $z<-1$, the inequality

$$
b^{s+1}<|a| \exp [-(s-1)(s \log s+(2 s+1) \log 2+s] .
$$

Then, we have

$$
\operatorname{dim}_{\mathbf{Q}}\left(\mathbf{Q}+\mathbf{Q} \operatorname{Li}_{1}(1 / z)+\cdots+\mathbf{Q} \operatorname{Li}_{s}(1 / z)\right)=s+1 .
$$

Key words and phrases: Pade approximation, irrationality, Lerch function. Received December 25, 2013.

Revised July 14, 2014. 
Let

$$
\Phi_{s}(x, z)=\sum_{m=0}^{\infty} \frac{z^{m+1}}{(m+x)^{s}} .
$$

be the Lerch function with $s \in \mathbf{N}, x>0$ and $z \in \mathbf{C}$ satisfying $|z| \leq 1$ and $(s, z) \neq$ $(1,1)$. The Lerch function is related to the Hurwitz zeta function $\zeta(s, x)$, the polylogarithm functions $\operatorname{Li}_{s}(z)$, and the Riemann zeta function $\zeta(s)$. In fact there are equations

$$
\zeta(s, x)=\Phi_{s}(x, 1), \quad \operatorname{Li}_{s}(z)=\Phi_{s}(1, z), \quad \zeta(s)=\Phi_{s}(1,1) .
$$

Rivoal [10] studied the Padé approximation of the Lerch function. In [10] Rivoal generalized the study of the Padé approximation of the polylogarithm functions in [9] and that of the Hurwitz zeta function in [2]. Rivoal remarked that the study of the Padé approximation of the Lerch function is related to the study of the Diophantine problems of the Riemann zeta function. In this paper, by using the Padé approximation of the Lerch function, Proposition 2.1, we prove the following generalization of the Nikisin's result, Theorem 0.1 , for the Lerch function. That is as follows

Theorem 0.2. Let $s \in \mathbf{N}$. Suppose that $x$ is a positive rational number and $z$ is a negative rational number and assume, for unique coprime integers $\alpha, \beta$ and $a, b$ such that $x=\alpha / \beta$ and $z=a / b$ with $\beta, b>0$ and $z<-1$, the inequality

$$
\begin{aligned}
b^{s+1}<|a| \exp ( & -\left[s^{2}\left(\log \beta+\sum_{q \mid \beta} \frac{\log q}{q-1}+\beta\right)\right. \\
& +(s-1)(s \log s+(2 s+1) \log 2)-s]),
\end{aligned}
$$

where $q$ runs through the prime divisors of $\beta$. Then, we have

$$
\operatorname{dim}_{\mathbf{Q}}\left(\mathbf{Q}+\mathbf{Q} \Phi_{1}(x, 1 / z)+\cdots+\mathbf{Q} \Phi_{s}(x, 1 / z)\right)=s+1 .
$$

REMARK 0.3. Note that Theorem 1 in [6] insists the same conclusion as the conclusion (2) of Theorem 0.1 with a condition

$$
b^{2}<|a| \exp [-(s-1)(s \log s+2 s \log 2)],
$$

which is weaker than the condition (1) in Theorem 0.1. Unfortunately, there are minor errors in the proof of Theorem 1 in [6]. More precisely, 
$B_{4} \exp \{n(s \ln s+2 s \ln 2)\} \quad$ on line 14, page 386 should be replaced by $B_{4} \exp \{n(s \ln s+(2 s+1) \ln s)\}$, and $b^{n} e^{s n}$ on line 11, page 388 should be replaced by $\left(b e^{s n}\right)^{s}$. These replacements lead the condition (1), which is the condition given in Theorem 0.2 with $\beta=1$.

REMARK 0.4. Hata evaluated the dimension of the Q-vector space spanned by some special values of the Lerch function in [5, Theorem 2.1]. His method is quite different from ours and our result is stronger than that of Hata's.

REMARK 0.5. It might be a natural question to ask the relation between the Padé approximation of Lerch function obtained in [10, Theorem 1] and that of ours (see Proposition 2.1 of this paper). At the moment, the relation is not clear to us.

\section{Acknowledgement}

The author would like to thank Professor Noriko Hirata for her constant encouragement and spending a lot of time to guide him to the theory of Diophantine approximation. The author is grateful to Professor Tadashi Ochiai for his constant encouragement and many comments on an earlier version of the manuscript, and to the referees for improving this paper.

\section{A Criterion of Linear Independence of Numbers}

In this section, we recall a criterion of linear independence for given real numbers. From now on throughout the paper, we fix a natural number $s \in \mathbf{N}$.

Lemma 1.1. Let $\theta_{1}, \ldots, \theta_{s} \in \mathbf{R}$. Suppose that there exist $(s+1)$ linear forms

$$
L_{q}^{(n)}\left(X_{0}, \ldots, X_{s}\right)=\sum_{k=0}^{s} A_{k, q}^{(n)} X_{k} \quad(0 \leq q \leq s ; n \in \mathbf{N}),
$$

for all $n \in \mathbf{N}$ with integer coefficients, which satisfy the following two conditions.

$$
\begin{aligned}
& \operatorname{det}\left(\left(A_{k, q}^{(n)}\right)_{0 \leq k, q \leq s}\right) \neq 0, \\
& \lim _{n \rightarrow \infty}\left(\max _{0 \leq q \leq s}\left\{\left|L_{q}^{(n)}(\boldsymbol{\theta})\right|\right\} \max _{0 \leq k, q \leq s}\left\{\left|A_{k, q}^{(n)}\right|\right\}^{s-1}\right)=0,
\end{aligned}
$$

where $L_{q}^{(n)}(\boldsymbol{\theta})=L_{q}^{(n)}\left(1, \theta_{1}, \ldots, \theta_{s}\right)$. Then, we have

$$
\operatorname{dim}_{\mathbf{Q}}\left(\mathbf{Q}+\mathbf{Q} \theta_{1}+\cdots+\mathbf{Q} \theta_{s}\right)=s+1 .
$$


Proof. We denote $\theta_{0}=1$. We consider a linear form with integer coefficients

$$
l=\sum_{i=0}^{s} x_{i} \theta_{i} \quad\left(\mathbf{x}:=\left(x_{0}, \ldots, x_{s}\right) \neq 0\right) .
$$

According to the condition (3), the determinant

$$
\Delta_{\mathbf{x}}^{(n)}:=\operatorname{det}\left(\begin{array}{cccc}
A_{0,0}^{(n)} & A_{1,0}^{(n)} & \cdots & A_{s, 0}^{(n)} \\
\vdots & \vdots & \ddots & \vdots \\
A_{0, p-1}^{(n)} & A_{1, p-1}^{(n)} & \cdots & A_{s, p-1}^{(n)} \\
x_{0} & x_{1} & \cdots & x_{s} \\
A_{0, p+1}^{(n)} & A_{1, p+1}^{(n)} & \cdots & A_{s, p+1}^{(n)} \\
\vdots & \vdots & \ddots & \vdots \\
A_{0, s}^{(n)} & A_{1, s}^{(n)} & \cdots & A_{s, s}^{(n)}
\end{array}\right) .
$$

is non zero for some $p$ which depends on $n$. Adding the $i$-th column multipled by $\theta_{i-1}$ for $2 \leq i \leq s+1$ to the first column, we get

$$
\Delta_{\mathbf{x}}^{(n)}=\operatorname{det}\left(\begin{array}{cccc}
L_{0}^{(n)}(\boldsymbol{\theta}) & A_{1,0}^{(n)} & \cdots & A_{s, 0}^{(n)} \\
\vdots & & \ddots & \vdots \\
L_{p-1}^{(n)}(\boldsymbol{\theta}) & A_{1, p-1}^{(n)} & \cdots & A_{s, p-1}^{(n)} \\
l & x_{1} & \cdots & x_{s} \\
L_{p+1}^{(n)}(\boldsymbol{\theta}) & A_{1, p+1}^{(n)} & \cdots & A_{s, p+1}^{(n)} \\
\vdots & & \ddots & \vdots \\
L_{s}^{(n)}(\boldsymbol{\theta}) & A_{1, s}^{(n)} & \cdots & A_{s, s}^{(n)}
\end{array}\right) .
$$

Since $\Delta_{\mathbf{x}}^{(n)} \neq 0$ and $\Delta_{\mathbf{x}}^{(n)} \in \mathbf{Z}$, we get $1 \leq\left|\Delta_{\mathbf{x}}^{(n)}\right|$ for all $n \in \mathbf{N}$. We estimate an upper bound of $\left|\Delta_{\mathbf{x}}^{(n)}\right|$. Let $\Delta_{\mathbf{x}, t, u}^{(n)}$ be the $(t, u)$-th cofactor of the above matrix. Then we have

$$
\Delta_{\mathbf{x}}^{(n)}=\sum_{t \neq p} L_{t}^{(n)}(\boldsymbol{\theta}) \Delta_{\mathbf{x}, t, 1}^{(n)}+l \Delta_{\mathbf{x}, p, 1}^{(n)}
$$

For each $t \neq p$ with $0 \leq t \leq s$, the definition of determinant implies,

$$
\left|\Delta_{\mathbf{x}, t, 1}^{(n)}\right| \leq s ! \max _{0 \leq k, q \leq s}\left\{\left|A_{k, q}^{(n)}\right|\right\}^{s-1} \max _{0 \leq q \leq s}\left\{\left|x_{q}\right|\right\} .
$$

Hence, we get

$$
\left|\Delta_{\mathbf{x}}^{(n)}\right| \leq \max _{0 \leq q \leq s}\left\{\left|L_{q}^{(n)}(\boldsymbol{\theta})\right|\right\} s ! \max _{0 \leq k, q \leq s}\left\{\left|A_{k, q}^{(n)}\right|\right\}^{s-1} \max _{0 \leq q \leq s}\left\{\left|x_{q}\right|\right\}+|l| s ! \max _{0 \leq k, q \leq s}\left\{\left|A_{k, q}^{(n)}\right|\right\}^{s} .
$$


From the condition (5), when $n$ tends to infinity, the first term of the right-hand side tends to zero. Thus we get $l \neq 0$. Thus we conclude that

$$
\operatorname{dim}_{\mathbf{Q}}\left(\mathbf{Q}+\mathbf{Q} \theta_{1}+\cdots+\mathbf{Q} \theta_{s}\right)=s+1
$$

REMARK 1.2. Lemma 1.1 is based on the Siegel's paper [11]. We gave here the detailed proof of it, following basically the argument given in [7, Chapter 2, Section 1.4].

\section{A Padé Approximation of the Lerch Function}

We would like to construct linear forms with integer coefficients satisfying the conditions (1), (2) of Lemma 1.1 for $\theta_{1}=\Phi_{1}(x, 1 / z), \ldots, \theta_{s}=\Phi_{s}(x, 1 / z)$ when $x, z \in \mathbf{Q}$ satisfy the conditions of Theorem 0.2 . For this purpose, we construct a Padé approximation of the Lerch function.

For a positive integer $n$ and non-negative integers $k, q$ with $0 \leq k, q \leq s$, we put

$$
\begin{aligned}
& F_{q}^{(n)}(x, u):=\frac{u(u-1) \cdots\left(u-\sigma_{n, q}+2\right)}{\prod_{j=0}^{n-1}(u+x+j)^{S}(u+x+n)^{q}}, \\
& \mathscr{R}_{q}^{(n)}(x, z):=\sum_{m=0}^{\infty} F_{q}^{(n)}(x, m) z^{-m-1},
\end{aligned}
$$

where $\sigma_{n, q}=n s+q$. Then we define a family of rational functions $\left\{c_{j, k, q}^{(n)}(x)\right\}_{1 \leq k \leq s, 0 \leq q \leq s, 0 \leq j \leq n}$ by

$$
F_{q}^{(n)}(x, u)=\sum_{k=1}^{s} \sum_{j=0}^{n} \frac{c_{j, k, q}^{(n)}(x)}{(u+x+j)^{k}}
$$

and a family of rational functions $\left\{A_{k, q}^{(n)}(x, z) \in \mathbf{Q}(x)[z]\right\}_{0 \leq k, q \leq s}$ by

(6) $\quad A_{k, q}^{(n)}(x, z)= \begin{cases}\sum_{j=0}^{n} c_{j, k, q}^{(n)}(x) z^{j} & \text { for } 1 \leq k \leq s, 0 \leq q \leq s \\ \sum_{p=1}^{s} \sum_{j=1}^{n} c_{j, p, q}^{(n)}(x) \sum_{l=0}^{j-1} \frac{z^{j-1-l}}{(x+l)^{p}} & \text { otherwise. }\end{cases}$

By the definition of $A_{k, q}^{(n)}(x, z)$, we can easily show

$$
\operatorname{deg}_{z} A_{k, q}^{(n)}(x, z) \leq \begin{cases}n-1 & \text { for } q=0 \\ n & \text { otherwise. }\end{cases}
$$


Using the family of rational functions $\left\{A_{k, q}^{(n)}(x, z)\right\}_{0 \leq k, q \leq s}$, we can construct a Padé approximation of the Lerch function.

Proposition 2.1. Under the notation above, we have $\mathscr{R}_{q}^{(n)}(x, z)=O\left(z^{-\sigma_{n, q}}\right)$ and the following Padé approximation

$$
\mathscr{R}_{q}^{(n)}(x, z)=\sum_{k=1}^{s} A_{k, q}^{(n)}(x, z) \Phi_{k}(x, 1 / z)-A_{0, q}^{(n)}(x, z) \quad \text { for } 0 \leq q \leq s .
$$

Further, we have the following integral representations

$$
\begin{gathered}
F_{q}^{(n)}(x, u)=\sum_{k=1}^{s} \int_{0}^{1} \frac{t^{x+u-1}}{\Gamma(k)} A_{k, q}^{(n)}(x, t)(\log 1 / t)^{k-1} d t \quad \text { for } 0 \leq q \leq s, \\
A_{0, q}^{(n)}(x, z)=\sum_{k=1}^{s} \int_{0}^{1} \frac{t^{x-1}}{\Gamma(k)} \frac{\left(A_{k, q}^{(n)}(x, z)-A_{k, q}^{(n)}(x, t)\right)}{z-t}(\log 1 / t)^{k-1} d t \\
\quad \text { for } 0 \leq q \leq s, \\
\mathscr{R}_{q}^{(n)}(x, z)=\sum_{k=1}^{s} \int_{0}^{1} \frac{t^{x-1}}{\Gamma(k)} \frac{A_{k, q}^{(n)}(x, t)}{z-t}(\log 1 / t)^{k-1} d t \quad \text { for } 0 \leq q \leq s,|z|>1 .
\end{gathered}
$$

Proof. Since the coefficients of $\mathscr{R}_{q}^{(n)}(x, z)$ of the expansion with respect to $z$ vanish for the degree larger than $-\sigma_{n, q}$ by the definition of $F_{q}^{(n)}(x, u)$, we get $\mathscr{R}_{q}^{(n)}(x, z)=O\left(z^{-\sigma_{n, q}}\right)$. By the relation (5), we get

$$
\begin{aligned}
\mathscr{R}_{q}^{(n)}(x, z) & =\sum_{m=0}^{\infty} \sum_{k=1}^{s} \sum_{j=0}^{n} \frac{c_{j, k, q}^{(n)}(x)}{(m+x+j)^{k}} z^{-m-1} \\
& =\sum_{k=1}^{s} \sum_{j=0}^{n} c_{j, k, q}^{(n)}(x)\left(\sum_{m=0}^{\infty} \frac{1}{(m+x+j)^{k}} z^{-m-1}\right) .
\end{aligned}
$$

Furthur we have the following equalities

$$
\begin{aligned}
\sum_{m=0}^{\infty} \frac{1}{(m+x+j)^{k}} z^{-m-1} & =\left(\sum_{m=0}^{\infty} \frac{1}{(m+x)^{k}} z^{-m-1}-\sum_{m=0}^{j-1} \frac{1}{(m+x)^{k}} z^{-m-1}\right) z^{j} \\
& =\left(\Phi_{k}(x, 1 / z)-\sum_{m=0}^{j-1} \frac{1}{(m+x)^{k}} z^{-m-1}\right) z^{j}
\end{aligned}
$$



Note that if $j=0$, we mean $\sum_{m=0}^{j-1} \frac{1}{(m+x)^{k}} z^{-m-1}=0$. By the above equalities,
we obtain

$$
\begin{aligned}
\mathscr{R}_{q}^{(n)}(x, z) & =\sum_{k=1}^{s} \sum_{j=0}^{n} c_{j, k, q}^{(n)}(x)\left(\Phi_{k}(x, 1 / z)-\sum_{m=0}^{j-1} \frac{1}{(m+x)^{k}} z^{-m-1}\right) z^{j} \\
& =\sum_{k=1}^{s}\left(\sum_{j=0}^{n} c_{j, k, q}^{(n)}(x) z^{j}\right) \Phi_{k}(x, 1 / z)-\sum_{k=1}^{s} \sum_{j=1}^{n} c_{j, k, q}^{(n)}(x) \sum_{m=0}^{j-1} \frac{1}{(m+x)^{k}} z^{-m+j-1} \\
& =\sum_{k=1}^{s} A_{k, q}^{(n)}(x, z) \Phi_{k}(x, 1 / z)-A_{0, q}^{(n)}(x, z) .
\end{aligned}
$$

Thus we obtain the equation (8).

Using the integration

$$
\int_{0}^{1} \frac{t^{x+u-1}}{\Gamma(k)}(\log 1 / t)^{k-1} d t=\frac{1}{(x+u)^{k}}
$$

the definition (5) and (6), we can show (9) as

$$
\begin{aligned}
F_{q}^{(n)}(x, u) & =\sum_{k=1}^{s} \sum_{j=0}^{n} \frac{c_{j, k, q}^{(n)}(x)}{(u+x+j)^{k}} \\
& =\sum_{k=1}^{s} \int_{0}^{1} \frac{t^{x+u-1}}{\Gamma(k)} A_{k, q}^{(n)}(x, t)(\log 1 / t)^{k-1} d t \quad \text { for } 0 \leq q \leq s .
\end{aligned}
$$

The integral representation (10) is obtained from the equation $\left(z^{j}-t^{j}\right) /(z-t)$ $=z^{j-1} \sum_{l=0}^{j-1}(t / z)^{l}$ and the above integration.

The integral representation (12) is obtained from the equation $\frac{1}{z-t}=$ $\sum_{m=0}^{\infty} t^{m} z^{-(m+1)}$ on $|t / z|<1$ and the above integration.

Remark 2.2. Let us keep the notation in Proposition 2.1. For $x \in \mathbf{Q} \backslash \mathbf{Z}_{\leq 0}$ and $q \in \mathbf{Z}_{\geq 1}$, the leading coefficient of the polynomial $A_{q, q}^{(n)}(x, z) \in \mathbf{Q}[z]$ is not zero for every $n \in \mathbf{N}$. In fact, we have

$$
\begin{aligned}
c_{n, q, q}^{(n)}(x) & =\left.\frac{u(u-1) \cdots\left(u-\sigma_{n, q}+2\right)}{\prod_{j=0}^{n-1}(u+x+j)^{s}(u+x+n)^{q}}(u+x+n)^{q}\right|_{u=-x-n} \\
& =\frac{(-x-n)(-x-n-1) \cdots\left(-x-n-\sigma_{n, q}+2\right)}{\prod_{j=0}^{n-1}(-n+j)^{s}} \neq 0 .
\end{aligned}
$$


Therefore we obtain

$$
\operatorname{deg}_{z}\left(A_{q, q}^{(n)}(x, z)\right)=n
$$

\section{Some Estimates}

By Propositin 2.1, we obtain linear systems with rational coeffitients of $1, \Phi_{1}(x, 1 / z), \ldots, \Phi_{s}(x, 1 / z)$ for $x, z \in \mathbf{Q}$. Multiplying a suitable integer to the coeffitients of each linear systems, we obtain a family of $(s+1)$ linear forms with integral coeffitients. To verify the conditions (1), (2) in Lemma 1.1 for the above linear forms, we would like to estimate some related values as a preparation for Section 5 .

LEMMA 3.1. Let $z$ and $x$ are real numbers satisfying $|z|>1$ and $x>0$. If $n \in \mathbf{N}$ is enough large, then there exists $c>0$ which is independent of $n$ and satisfies

$$
\max _{0 \leq k, q \leq s}\left\{\left|A_{k, q}^{(n)}(x, z)\right|\right\} \leq|z|^{n} n^{c} \exp \{n(s \log s+(2 s+1) \log 2)\} .
$$

ProOF. Using the definition of $\left\{c_{j, k, q}^{(n)}(x)\right\}_{1 \leq k \leq s, 0 \leq q \leq s, 0 \leq j \leq n}$ given by (5), we get

$$
c_{j, k, q}^{(n)}(x)=\frac{1}{2 \pi i} \int_{|u+j+x|=1 / 2} F_{q}^{(n)}(x, u)(u+x+j)^{k-1} d u .
$$

Using the integral representation of $c_{j, k, q}^{(n)}(x)$ and the definition of $F_{q}^{(n)}(x, u)$, we obtain

$$
\begin{aligned}
\left|c_{j, k, q}^{(n)}(x)\right| & \leq 2^{-k} \sup _{|u+j+x|=1 / 2}\left\{\left|F_{q}^{(n)}(x, u)\right|\right\} \\
& \leq 2^{-k} \sup _{|u+j+x|=1 / 2}\left|\frac{\left(u+\sigma_{n, q}-2\right)_{\sigma_{n, q}-1}}{(u+x)_{n}^{s}(u+n+x)^{q}}\right| .
\end{aligned}
$$

We give an upper bound of $\sup _{|u+j+x|=1 / 2}\left|\frac{\left(u-\sigma_{n, q}+2\right)_{\sigma_{n, q}-1}}{(u+x)_{n}^{s}(u+n+x)^{q}}\right|$. There are the following inequalities for $u \in\left\{u \in \mathbf{C}|| u+j+x \mid=\frac{1}{2}\right\}$,

$$
\begin{aligned}
\left|\left(u-\sigma_{n, q}+2\right)_{\sigma_{n, q}-1}\right| & =\left|(u+j+x-j-x) \cdots\left(u+j+x-\sigma_{n, q}-j-x+2\right)\right| \\
& \leq(1 / 2+j+x) \cdots\left(1 / 2+\sigma_{n, q}+j+x-2\right) \\
& \leq(1+j+[x]) \cdots\left(\sigma_{n, q}+j+[x]-1\right),
\end{aligned}
$$




$$
\begin{aligned}
|u+x|_{n} & =\prod_{l=0}^{n-1}|u+l+x|=\prod_{l=0}^{n-1}|u+j+x+(l-j)| \\
& \geq \prod_{l=0}^{n-1}|| l-j\left|-\frac{1}{2}\right| \geq \frac{(n-j) ! j !}{2^{3} n^{3}}
\end{aligned}
$$

and

$$
|u+n+x|=|| u+j+x|-| j-n|| \geq 1 / 2,
$$

where $[x] \in \mathbf{Z}$ satisfying $x-1<[x] \leq x$. We obtain

$$
\begin{aligned}
2^{-k} \sup _{|u+j+x|=1 / 2}\left|\frac{\left(u-\sigma_{n, q}+2\right)_{\sigma_{n, q}-1}}{(u+x)_{n}^{s}(u+n+x)^{q}}\right| \\
\quad \leq 2^{-k} \frac{(1+j+[x]) \cdots\left(\sigma_{n, q}+j+[x]-1\right) 2^{3 s+q} n^{3 s}}{((n-j) ! j !)^{s}} \\
\quad=2^{3 s+q-k} n^{3 s} \frac{\left(j+\sigma_{n, q}+[x]-1\right) !}{(j+[x]) !(n !)^{s}}\left(\begin{array}{c}
n \\
j
\end{array}\right)^{s}
\end{aligned}
$$

By using the inequality $\left(\begin{array}{l}n \\ j\end{array}\right) \leq 2^{n}$, we get

$$
\begin{aligned}
2^{3 s+q-k} n^{3 s} \frac{\left(j+\sigma_{n, q}+[x]-1\right) !}{(j+[x]) !(n) !)^{s}}\left(\begin{array}{c}
n \\
j
\end{array}\right)^{s} & \leq 2^{3 s+q-k} n^{3 s} \frac{\left(j+\sigma_{n, q}+[x]-1\right) !}{(j+[x]) !(n !)^{s}} 2^{n s} \\
& \leq 2^{3 s+q-k+j+\sigma_{n, q}+[x]-1} n^{3 s} \frac{\left(\sigma_{n, q}-1\right) !}{(n !)^{s}} 2^{n s}
\end{aligned}
$$

By using the Stirling formula, we obtain

$$
\begin{aligned}
2^{3 s+q-k+j+\sigma_{n, q}+[x]-1} n^{3 s} \frac{\left(\sigma_{n, q}-1\right) !}{(n !)^{s}} 2^{n s} & \leq n^{c^{\prime \prime}} \frac{\left(\sigma_{n, q}-1\right)^{\left(\sigma_{n, q}-1\right)} e^{-\left(\sigma_{n, q}-1\right)}}{n^{n s} e^{-n s}} 2^{2 n s+n} \\
& \leq n^{c^{\prime}} \exp \{n(s \log s+(2 s+1) \log 2)\}
\end{aligned}
$$

where $c^{\prime}, c^{\prime \prime}>0$ do not depend on $n$. From the above inequalities, we conclude that

$$
\left|c_{j, k, q}^{(n)}(x)\right| \leq n^{c^{\prime}} \exp \{n(s \log s+(2 s+1) \log 2)\} .
$$


Using the assumption $x>0,|z|>1$, the definition (6) and the above inequality, we obtain

$$
\left|A_{k, q}^{(n)}(x, z)\right| \leq \begin{cases}n^{c^{\prime}} \exp \{n(s \log s+(2 s+1) \log 2)\} \frac{|z|^{n+1}-1}{|z|-1} & \text { for } 1 \leq k \leq s \\ n^{c^{\prime}} \exp \{n(s \log s+(2 s+1) \log 2)\}|z|^{n-1} x \frac{x^{s}-1}{x-1} & \text { otherwise }\end{cases}
$$

for some $c^{\prime}>0$ which does not depend on $n$. Thus we conclude that

$$
\max _{0 \leq k, q \leq s}\left\{\left|A_{k, q}^{(n)}(x, z)\right|\right\} \leq|z|^{n} n^{c} \exp \{n(s \log s+(2 s+1) \log 2)\}
$$

for some $c>0$ which does not depend on $n$.

LEMma 3.2. Let $x, z$ are real numbers which satisfy $x>1 / 2, z<-1$. If $n \in \mathbf{N}$ is enough large, then there exists $c>0$ which is independent of $n$ and satisfies

$$
\max _{0 \leq q \leq s}\left\{\left|\mathscr{R}_{q}^{(n)}(x, z)\right|\right\} \leq n^{c}\left(\frac{1}{|z|}\right)^{n s}\left(1+\frac{1}{s}\right)^{-n s(s+1)}
$$

Proof. We remark that if $|x / z|<1$ and $x \neq 0$, then we have the following equation

$$
\frac{1}{z-t}=\frac{1}{2 i z} \int_{\operatorname{Re}(w)=-1 / 2}\left(-\frac{t}{z}\right)^{w} \frac{d w}{\sin (\pi w)} .
$$

The above equation is easily computed by means of residues.

Since $0<t \leq 1, \quad z<-1$, the integral converges uniformly for $t \in[\varepsilon, 1]$, $z<-1-\varepsilon$ where $0<\varepsilon<1$, we obtain

$$
\begin{aligned}
\mathscr{R}_{q}^{(n)}(x, z) & =\sum_{k=1}^{s} \int_{0}^{1} \frac{t^{x-1}}{\Gamma(k)} \frac{A_{k, q}^{(n)}(x, t)}{z-t}(\log 1 / t)^{k-1} d t \\
& =\frac{1}{2 i z} \int_{\operatorname{Re}(w)=-1 / 2} \sum_{k=1}^{s} \int_{0}^{1} \frac{t^{x-1}}{\Gamma(k)} A_{k, q}^{(n)}(x, t)(\log 1 / t)^{k-1} d t\left(-\frac{t}{z}\right)^{w} \frac{d w}{\sin (\pi w)} \\
& =\frac{1}{2 i z} \int_{\operatorname{Re}(w)=-1 / 2} F_{q}^{(n)}(x, w)\left(-\frac{1}{z}\right)^{w} \frac{d w}{\sin (\pi w)} .
\end{aligned}
$$

The last equality was obtained from the equality (9). Shifting the line of integration from $\operatorname{Re}(w)=-1 / 2$ to $\operatorname{Re}(w)=\sigma_{n, q}-3 / 2$, we get

$$
\mathscr{R}_{q}^{(n)}(x, z)=\frac{1}{2 i z} \int_{\operatorname{Re}(w)=\sigma_{n, q}-3 / 2} F_{q}^{(n)}(x, w)\left(-\frac{1}{z}\right)^{w} \frac{d w}{\sin (\pi w)} .
$$


We fix $v \in \mathbf{R}$ and put $w=\sigma_{n, q}-3 / 2+i v$. Then we get

$$
\begin{aligned}
& \left|F_{q}^{(n)}(x, w)\left(-\frac{1}{z}\right)^{w} \frac{1}{\sin (\pi w)}\right| \\
& \quad \leq \frac{A_{1}}{|z|^{\sigma_{n, q}}}\left|\frac{\Gamma(w+1)}{\Gamma\left(w-\sigma_{n, q}+2\right) \sin (\pi w)}\right|\left|\frac{1}{(w+x)^{s} \cdots(w+n-1+x)^{s}}\right| \\
& \quad \leq \frac{A_{2}}{|z|^{\sigma_{n, q}}} \Gamma\left(\sigma_{n, q}\right) \frac{1}{\left(\sigma_{n, q}+1\right)^{s} \cdots\left(\sigma_{n, q}+n\right)^{s}} .
\end{aligned}
$$

The third inequality is obtained as follows. Since there is the equality

$$
\frac{1}{\Gamma\left(w-\sigma_{n, q}+2\right) \sin (\pi w)}=\frac{\Gamma\left(\sigma_{n, q}-1-w\right)}{\pi} \text { for } w \in \mathbf{C} \backslash \mathbf{Z},
$$

we can obatine the inequality for $w=\sigma_{n, q}-3 / 2+i v$

$$
\left|\frac{\Gamma(w+1)}{\Gamma\left(w-\sigma_{n, q}+2\right) \sin (\pi w)}\right| \leq \Gamma\left(\sigma_{n, q}\right) \text {. }
$$

By using Stirling formula, we get

$$
\begin{aligned}
& \frac{A_{2}}{|z|^{\sigma_{n, q}}} \Gamma\left(\sigma_{n, q}\right) \frac{1}{\left(\sigma_{n, q}+1\right)^{s} \cdots\left(\sigma_{n, q}+n\right)^{s}} \\
& \quad \leq \frac{A_{3}}{|z|^{\sigma_{n, q}}} \sigma_{n, q}^{\sigma_{n, q}} e^{-\sigma_{n, q}} \frac{\sigma_{n, q}^{s \sigma_{n, q}} e^{-s \sigma_{n, q}}}{\left(\sigma_{n, q}+n\right)^{\left(\sigma_{n, q}+n\right)} e^{-s\left(\sigma_{n, q}+n\right)}} \\
& \quad \leq \frac{A_{4}}{|z|^{\sigma_{n, q}}}\left(1+\frac{1}{s}\right)^{-n s(s+1)} .
\end{aligned}
$$

for $A_{1}, A_{2}, A_{3}, A_{4}=\mathcal{O}\left(n^{c}\right)$ and some $c>0$ which does not depend on $n$. Thus we obtain the required estimate.

We denote

$$
d_{n}=1 . \mathrm{c} \cdot \mathrm{m} \cdot(1,2, \ldots, n)
$$

and

$$
\mu_{n}(b)=\prod_{q \mid b} q^{[n /(q-1)]}
$$

where $b, n \in \mathbf{N}$ and the product is over all primes $q$ dividing $b$. 
Lemma 3.3. Let $x$ and $z$ be rational numbers which satisfy $x>0$ and $z<-1$. Set $x=\frac{\alpha}{\beta}$ and $z=\frac{a}{b}$, where $\alpha, \beta$ with $\beta>0$ and $a, b$ with $b>0$ are coprime integers, respectively. Then we have

$$
\begin{aligned}
& s ! \beta^{n s+s-1} \mu_{n}(\beta)^{s} b^{n} d_{n}^{s-k} A_{k, q}^{(n)}(x, z) \in \mathbf{Z} \text { for } 1 \leq k \leq s, 0 \leq q \leq s, \\
& s ! \beta^{n s+s-1} \mu_{n}(\beta)^{s} b^{n}\left(d_{\alpha+(n-1) \beta}\right)^{s} A_{0, q}^{(n)}(x, z) \in \mathbf{Z} \quad \text { for } 0 \leq q \leq s .
\end{aligned}
$$

Especially we get

$$
s ! \beta^{n s+s-1} \mu_{n}(\beta)^{s} b^{n}\left(d_{\alpha+(n-1) \beta}\right)^{s} A_{k, q}^{(n)}(x, z) \in \mathbf{Z} \quad \text { for } 0 \leq k, q \leq s .
$$

Proof. We construct an integer which is divisible by the denominator of $c_{j, k, q}^{(n)}(x)$ for $0 \leq k, q \leq s, 0 \leq j \leq n$. According to the equation (3), we get

$$
c_{j, k, q}^{(n)}(x)= \begin{cases}\left.\frac{1}{(s-k) !}\left(\frac{d}{d u}\right)^{s-k} F_{q}^{(n)}(x, u)(u+x+j)^{s}\right|_{u=-x-j} & \text { for } 0 \leq j \leq n-1, \\ \left.\frac{1}{(q-k) !}\left(\frac{d}{d u}\right)^{q-k} F_{q}^{(n)}(x, u)(u+x+n)^{q}\right|_{u=-x-n} & \text { for } j=n \\ 0 & 1 \leq k \leq q \\ & \text { for } j=n, k>q .\end{cases}
$$

First we caluculate $\left.\frac{1}{(s-k) !}\left(\frac{d}{d u}\right)^{s-k} F_{q}^{(n)}(x, u)(u+x+j)^{s}\right|_{u=-x-j}$ for $0 \leq j \leq$ $n-1,1 \leq k \leq s$. Since we have the following equality

$$
\begin{aligned}
F_{q}^{(n)} & (x, u)(u+x+j)^{s} \\
& =\frac{u(u-1) \cdots\left(u-\sigma_{n, q}+2\right)}{(u+x)^{s} \cdots(u+x+j-1)^{s}(u+x+j+1)^{s} \cdots(u+x+n-1)^{s}(u+x+n)^{q}},
\end{aligned}
$$

$F_{q}^{(n)}(x, u)(u+x+j)^{s}$ can be represented in the form of a product of the following functions.

$$
\begin{aligned}
& P_{q}^{(n)}(u)=\left(u-\sigma_{n, q}+2\right)\left(u-\sigma_{n, q}+3\right) \cdots\left(u-\sigma_{n, q}+s\right), \\
& F_{c, q, n}(x, u) \\
& =\frac{(u-c)(u-c-1) \cdots(u-c-(n-1))}{(u+x)(u+x+1) \cdots(u+x+j-1)(u+x+j+1) \cdots(u+x+n)} \quad \text { for } c \geq 0 . \\
& F_{c, q, n-1}(x, u) \\
& =\frac{(u-c)(u-c-1) \cdots(u-c-(n-2))}{(u+x)(u+x+1) \cdots(u+x+j-1)(u+x+j+1) \cdots(u+x+n-1)}
\end{aligned}
$$


We denote

$$
F_{q}^{(n)}(x, u)(u+x+j)^{s}:=P_{q}^{(n)}(u) \prod_{m=1}^{s} F_{c_{m}, q}^{(n)}(x, u),
$$

where $F_{c_{m}, q}^{(n)}(x, z)$ stands for either $F_{c, q, n}(x, u)$ or $F_{c, q, n-1}(x, u)$. Hence, we get

$$
\begin{aligned}
& \left.\left(\frac{d}{d u}\right)^{s-k} F_{q}^{(n)}(x, u)(u+x+j)^{s}\right|_{u=-j-x} \\
& \left.\quad=\left.\sum_{l_{0}+l_{1}+\cdots+l_{s}=s-k} \frac{(s-k) !}{l_{0} ! \cdots l_{s} !}\left(\left(\frac{d}{d u}\right)^{l_{0}} P_{q}^{(n)}(u) \prod_{m=1}^{s}\left(\frac{d}{d u}\right)^{l_{m}} F_{c_{m}, q}^{(n)}(x, u)\right)\right|_{u=-j-x}\right) .
\end{aligned}
$$

We can express

$$
\begin{aligned}
F_{c, q, n}(x, u)= & \frac{(u-c)(u-c-1) \cdots(u-c-(n-1))}{(u+x)(u+x+1) \cdots(u+x+j-1)(u+x+j+1) \cdots(u+x+n)} \\
= & 1+\frac{B_{0, q}(x) j}{(u+x)}+\frac{B_{1, q}(x)(j-1)}{(u+x+1)}+\cdots \frac{B_{j-1, q}(x)}{(u+x+j-1)} \\
& +\frac{B_{j+1, q}(x)}{(u+x+j+1)}+\cdots+\frac{B_{n, q}(x)(n-j)}{(u+x+n)}
\end{aligned}
$$

where

$$
B_{l, q}(x)= \begin{cases}(-1)^{n+l} \frac{(x+l+c) \cdots(x+c+l+n-1)}{l !(n-l) !} & \text { for } 0 \leq l \leq j \\ (-1)^{n+l-1} \frac{(x+l+c) \cdots(x+c+l+n-1)}{l !(n-l) !} & \text { for } j+1 \leq l \leq n .\end{cases}
$$

Substituting $\alpha / \beta$ for $x$, we get

$$
B_{l, q}(x)= \begin{cases}\frac{(-1)^{n+l} \beta^{-n} \prod_{w=0}^{n-1}(\alpha+\beta(c+l+w))}{l !(n-l) !} & \text { for } 0 \leq l \leq j \\ \frac{(-1)^{n+l-1} \beta^{-n} \prod_{w=0}^{n-1}(\alpha+\beta(c+l+w))}{l !(n-l) !} & \text { for } j+1 \leq l \leq n .\end{cases}
$$

Since

$$
\frac{\beta^{-n} \prod_{w=0}^{n-1}(\alpha+\beta(c+l+w))}{l !(n-l) !}=\frac{\beta^{-n} \prod_{w=0}^{n-1}(\alpha+\beta(c+l+w))}{n !} \frac{n !}{l !(n-l) !}
$$

and $(\alpha, \beta)=1$, we obtain $\mu_{n}(\beta) \frac{\prod_{w=0}^{n-1}(\alpha+\beta(c+l+w))}{n !} \in \mathbf{Z}$. Henceforce we get

$$
\mu_{n}(\beta) \beta^{n} B_{l, q}(x) \in \mathbf{Z} \quad \text { for } 0 \leq q \leq s, n \in \mathbf{N} .
$$


Using the above relation and the following equation

$$
\begin{aligned}
\left(\frac{d}{d u}\right)^{l}\left(F_{c, q, n}(x, u)\right) & \\
= & 1^{(l)}+(-1)^{l} l !\left[\frac{B_{0, q}(x) j}{(u+x)^{l+1}}+\frac{B_{1, q}(x)(j-1)}{(u+x+1)^{l+1}}+\cdots+\frac{B_{j-1, q}(x)}{(u+x+j-1)^{l+1}}\right. \\
& \left.+\frac{B_{j+1, q}(x)}{(u+x+j+1)^{l+1}}+\cdots+\frac{B_{n, q}(x)(n-j)}{(u+x+n)^{l+1}}\right] \quad \text { for } 0 \leq l \leq s-k,
\end{aligned}
$$

we obtain

$$
\begin{aligned}
\left.\mu_{n}(\beta) \beta^{n} d_{n}^{l}\left(\frac{d}{d u}\right)^{l}\left(F_{c, q, n}(x, u)\right)\right|_{u=-j-x} \\
=\mu_{n}(\beta) \beta^{n} d_{n}^{l}\left[1^{(l)}+(-1)^{l} l !\left[\frac{(-1)^{l+1} B_{0, q}(x)}{j^{l}}+\frac{(-1)^{l+1} B_{1, q}(x)}{(j-1)^{l}} \cdots\right.\right. \\
\left.\left.\quad+(-1)^{l+1} B_{j-1, q}(x)+B_{j+1, q}(x)+\cdots+\frac{B_{n, q}(x)(n-j)}{(n-j)^{l}}\right]\right] \in \mathbf{Z} .
\end{aligned}
$$

Similarly, we obtain

$$
\left.\mu_{n-1}(\beta) \beta^{n-1} d_{n-1}^{l}\left(\frac{d}{d_{u}}\right)^{l}\left(F_{c, q, n-1}(x, u)\right)\right|_{u=-j-x} \in \mathbf{Z} .
$$

Thus we obtain

$$
s ! \mu_{n}(\beta)^{s} \beta^{n s+s-1} d_{n}^{s-k} c_{k, j, q}^{(n)}(x) \in \mathbf{Z} .
$$

We conclude that

$$
s ! \mu_{n}(\beta)^{s} \beta^{n s+s-1} b^{n} d_{n}^{s-k} A_{k, q}^{(n)}(x, z) \in \mathbf{Z} .
$$

Next we consider the case of $k=0$. From the definition (6) we get the equation

$$
\begin{aligned}
A_{0, q}^{(n)}(x, z) & =\sum_{k=1}^{s} \sum_{j=1}^{n} c_{j, k, q}^{(n)}(x)\left(\frac{z^{j-1}}{x^{k}}+\frac{z^{j-2}}{(x+1)^{k}}+\cdots+\frac{1}{(x+j-1)^{k}}\right) \\
& =\sum_{k=1}^{s} \sum_{j=1}^{n} c_{j, k, q}^{(n)}(x)\left(\frac{a^{j-1} \beta^{k}}{b^{j-1} \alpha^{k}}+\frac{a^{j-2} \beta^{k}}{b^{j-2}(\alpha+\beta)^{k}}+\cdots+\frac{\beta^{k}}{(\alpha+(j-1) \beta)^{k}}\right) .
\end{aligned}
$$


Since $s ! \mu_{n}(\beta)^{s} \beta^{n s+s-1} d_{n}^{s-k} c_{j, k, q}^{(n)}(x) \in \mathbf{Z}$, we conclude that

$$
s ! \mu_{n}(\beta)^{s} \beta^{n s+s-1} b^{n}\left(d_{\alpha+(n-1) \beta}\right)^{s} A_{0, q}^{(n)}(x, z) \in \mathbf{Z} .
$$

We put

$$
\Delta^{(n)}(x, z)=\operatorname{det}\left(\begin{array}{cccc}
-A_{0,0}^{(n)}(x, z) & A_{1,0}^{(n)}(x, z) & \cdots & A_{s, 0}^{(n)}(x, z) \\
\vdots & \vdots & \ddots & \vdots \\
-A_{0, s}^{(n)}(x, z) & A_{1, s}^{(n)}(x, z) & \cdots & A_{s, s}^{(n)}(x, z)
\end{array}\right)
$$

for every $n \in \mathbf{N}$. Then we have the following lemma

Lemma 3.4. Let $\Delta^{(n)}(x, z)$ be as above. Then $\Delta^{(n)}(x, z)$ are non-zero polynomials with rational coefficients in variable $x$ for every $n \in \mathbf{N}$.

Proof. We denote

$$
\begin{array}{r}
\mathscr{R}_{q}^{(n)}(x, z):=\sum_{k=1}^{s} A_{k, q}^{(n)}(x, z) \Phi_{k}(x, z)-A_{0, q}^{(n)}(x, z)=\frac{c_{0, q}^{(n)}(x)}{z^{\sigma_{n, q}}}+\cdots, \\
\Delta_{q}^{(n)}(x, z):=(-1)^{s+q} \operatorname{det}\left(\begin{array}{ccc}
A_{1,0}^{(n)}(x, z) & \cdots & A_{s, 0}^{(n)}(x, z) \\
\vdots & \ddots & \vdots \\
A_{1, q-1}^{(n)}(x, z) & \cdots & A_{s, q-1}^{(n)}(x, z) \\
A_{1, q+1}^{(n)}(x, z) & \cdots & A_{s, q+1}^{(n)}(x, z) \\
\vdots & \ddots & \vdots \\
A_{1, s}^{(n)}(x, z) & \cdots & A_{s, s}^{(n)}(x, z)
\end{array}\right),
\end{array}
$$

and

$$
\Delta^{(n)}(x, z)=\operatorname{det} A^{(n)}(x, z)=\operatorname{det}\left(\begin{array}{cccc}
\mathscr{R}_{0}^{(n)}(x, z) & A_{1,0}^{(n)}(x, z) & \cdots & A_{s, 0}^{(n)}(x, z) \\
\vdots & \vdots & \ddots & \vdots \\
\mathscr{R}_{s}^{(n)}(x, z) & A_{1, s}^{(n)}(x, z) & \cdots & A_{s, s}^{(n)}(x, z)
\end{array}\right) .
$$

We consider $\Delta_{q}^{(n)}(x, z)$ as a polynomial in $z$. From the construction of $A_{k, q}^{(n)}(x, z)$ we get

$$
\operatorname{deg}_{z} \Delta_{q}^{(n)}(x, z) \begin{cases}\leq(n-1)+n+\cdots+n=n s-1 & \text { for } q \geq 1 \\ =n s & \text { for } q=0\end{cases}
$$


When $q=0$, we use the equation (12) in Remark 2.2. If we denote

$$
b^{(n)}(x):=\prod_{q=1}^{s} c_{n, q, q}^{(n)}(x),
$$

then, we get

$$
\begin{aligned}
\Delta^{(n)}(x, z) & =\sum_{q=0}^{s}-A_{0, q}^{(n)}(x, z) \Delta_{q}^{(n)}(x, z) \\
& =\sum_{q=0}^{s} \mathscr{R}_{q}^{(n)}(x, z) \Delta_{q}^{(n)}(x, z) \\
& =b^{(n)}(x) c_{0,0}^{(n)}(x)+h_{1}(x) / z+h_{2}(x) / z^{2}+\cdots .
\end{aligned}
$$

Since $\Delta^{(n)}(x, z)$ is a polynomial in $x$ and $z$, we get

$$
\Delta^{(n)}(x, z)=b^{(n)}(x) c_{0,0}^{(n)}(x) \in \mathbf{Q}[x] .
$$

Moreover, since $c_{0,0}^{(n)}(x)$ is not zero, $\Delta^{(n)}(x, z) \neq 0$.

REMARK 3.5. By Remark 2.2 and the equation (17) in Lemma 3.3, we have $b^{(n)}(x) \neq 0$ and $c_{0,0}(x) \neq 0$ for $x \in \mathbf{Q}_{>0}$.

\section{Proof of the Main Theorem}

In this section, $x$ and $z$ are rational numbers satisfying the condition of Theorem 0.2. Theorem 0.2 is proved by using the result of Sections 2, 3 and 4 . We construct $(s+1)$ linear forms with integer coefficients satisfying the conditions (1), (2) in Lemma 1.1 for $\Phi_{1}(x, 1 / z), \ldots, \Phi_{s}(x, 1 / z)$. We use the notations of the previous section.

We put

$$
A_{k, q}^{(n)}:=s ! \beta^{n s+s-1} \mu_{n}(\beta)^{s} b^{n}\left(d_{\alpha+(n-1) \beta}\right)^{s} A_{k, q}^{(n)}(x, z) \text { for } 0 \leq k, q \leq s,
$$

and

$$
L_{q}^{(n)}\left(X_{0}, \ldots, X_{s}\right):=-A_{0, q}^{(n)} X_{0}+\sum_{k=1}^{s} A_{k, q}^{(n)} X_{q} \text { for } 0 \leq q \leq s, n \in \mathbf{N}
$$

By the equation (16) in Lemma 3.3, $\left\{L_{q}^{(n)}\left(X_{0}, \ldots, X_{s}\right)\right\}_{0 \leq q \leq s}$ are the linear forms with integer coefficients for all $n \in \mathbf{N}$. 
We denote

$$
\Delta^{(n)}=\operatorname{det}\left(\begin{array}{ccc}
-A_{0,0}^{(n)} & \cdots & A_{0, s}^{(n)} \\
\vdots & \ddots & \vdots \\
-A_{s, 0}^{(n)} & \cdots & A_{s, s}^{(n)}
\end{array}\right)=\operatorname{det}\left(\begin{array}{ccc}
-L_{0}^{(n)}(\boldsymbol{\theta}) A_{0,1}^{(n)} & \cdots & A_{0, s}^{(n)} \\
\vdots & \ddots & \vdots \\
-L_{s}^{(n)}(\boldsymbol{\theta}) A_{s, 1}^{(n)} & \cdots & A_{s, s}^{(n)}
\end{array}\right)
$$

Now we are ready to prove Theorem 0.2 .

Proof. By Lemma 3.4 and Remark 3.5, we get $\Delta^{(n)} \neq 0$ for all $n \in \mathbf{N}$. By the inequalities (13), (15) in Lemma 3.1 and 3.2 respectively, we get

$$
\begin{aligned}
& \max _{0 \leq q \leq s}\left\{\left|L_{q}^{(n)}(\boldsymbol{\theta})\right|\right\} \max _{0 \leq k, q \leq s}\left\{\left|A_{k, q}^{(n)}\right|\right\}^{s-1} \\
& \leq A_{1}\left\{\beta^{n s+s-1} \mu_{n}(\beta)^{s} b^{n}\left(d_{\alpha+(n-1) \beta}\right)^{s}\right\}^{s}\left(\frac{1}{|z|}\right)^{n s}\left(1+\frac{1}{s}\right)^{-n s(s+1)} \\
&|z|^{n(s-1)} \exp \{(s-1)(s \log s+(2 s+1) \log 2)\}^{n} .
\end{aligned}
$$

where $A_{1}=\mathcal{O}\left(n^{c}\right)$ for some $c>0$ which does not depend on $n$. Using the fact $d_{n}=\exp \{n+o(n)\}$ and $e \leq(1+1 / s)^{s+1}$ we get

$$
\begin{gathered}
\max _{0 \leq q \leq s}\left\{\left|L_{q}^{(n)}(\boldsymbol{\theta})\right|\right\} \max _{0 \leq k, q \leq s}\left\{\left|A_{k, q}^{(n)}\right|\right\}^{s-1} \\
\leq A_{2}\left(\beta^{n s} \mu_{n}(\beta)^{s} b^{n} e^{(\alpha+(n-1) \beta) s}\right)^{s} e^{-n s}\left(\frac{1}{|z|}\right)^{n} \exp \{(s-1)(s \log s+(2 s+1) \log 2)\}^{n} \\
\leq A_{3}\left[( \frac { b ^ { s + 1 } } { | a | } ) \operatorname { e x p } \left(s^{2}\left(\log \beta+\sum_{q \mid \beta} \frac{\log q}{q-1}+\beta\right)\right.\right. \\
+(s-1)(s \log s+(2 s+1) \log 2)-s))]^{n}
\end{gathered}
$$

where $A_{2}, A_{3}=\exp (o(n))$. Suppose

$$
\begin{aligned}
b^{s+1}<|a| \exp ( & -\left[s^{2}\left(\log \beta+\sum_{q \mid \beta} \frac{\log q}{q-1}+\beta\right)\right. \\
& +(s-1)(s \log s+(2 s+1) \log 2)-s)]),
\end{aligned}
$$


then,

$$
\max _{0 \leq q \leq s}\left\{\left|L_{q}^{(n)}(\boldsymbol{\theta})\right|\right\} \max _{0 \leq k, q \leq s}\left\{\left|A_{k, q}^{(n)}\right|\right\}^{s-1}
$$

goes to 0 when $n$ tends to infinity. By Lemma 1.1, we conclude that

$$
\operatorname{dim}_{\mathbf{Q}}\left(\mathbf{Q}+\mathbf{Q} \Phi_{1}(x, 1 / z)+\cdots+\mathbf{Q} \Phi_{s}(x, 1 / z)\right)=s+1 .
$$

\section{References}

[1] F. Beukers, Padé-approximations in number theory. In: Padé Approximation and Its Applications, Lecture Notes in Math. 888 Springer, Berlin, (1981), pp. 90-99.

[2] F. Beukers, Irrationality of some p-adic L-values, Acta Math. Sin, 24-4, (2008), pp. 663-686.

[3] K. Ball and T. Rivoal, Irrationalité d'une infinité de valeurs de la fonction zêta aux entiers impairs, Invent. Math. 146.1 (2001), pp. 193-207.

[4] S. Fischler and T. Rivoal, Approximants de Padé et séries hypergéométriques équilibrées, J. Math. Pures Appl. 82 (2003), no. 10, pp. 1369-1394.

[5] M. Hata, On the liner independence of the values of polylogarithmic functions, J. Math. Pures Appl. 69 (1990), pp. 133-173.

[6] E. M. Nikisin, On irrationality of the values of the functions $F(x, s)$, Math. USSR Sbornik. Vol. 37 (1980), No. 3, pp. 381-388.

[7] N. I. Fel'dman and Yu. V. Nesterenko, Number Theory IV, Transcendental Numbers, Encyclopaedia of Mathematical Science 44, Springer-Verlag, Berlin, (1998).

[8] M. Prévost, A new proof of the irrationality of $\zeta(2)$ and $\zeta(3)$ using Padé approximants, J. Comp. Appl. Math, 67 (1996), pp. 219-235.

[9] T. Rivoal, La fonction zêta de Riemann prend une infinité de valeurs irrationnelles aux entiers impairs, C. R. Acad. Sci. Paris Sér. I Math. 331 no. 4 (2000), pp. 267-270.

[10] T. Rivoal, Simultaneous polynomial approximations of the Lerch function, Canad. J. Math. 61 (6), 2009, pp. 1341-1356.

[11] C. Siegel, Uber einige Anwendungen diophantischer Approximationen, Abhandlungen der Preu $\beta$ ischen Akademie der Wissenschaften. Physikalisch-mathematische Kalasse 1929, Nr.1.

Department of Mathematics

Graduate School of Science, Osaka University

1-1 Machikaneyama, Toyonaka, Osaka 560-0043

Japan

E-mail: KawashimaMakotou784829k@ecs.osaka-u.ac.jp 